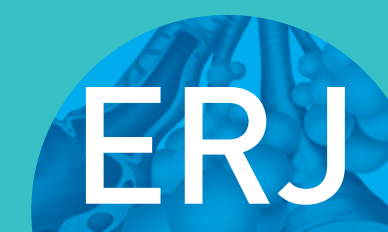

open research
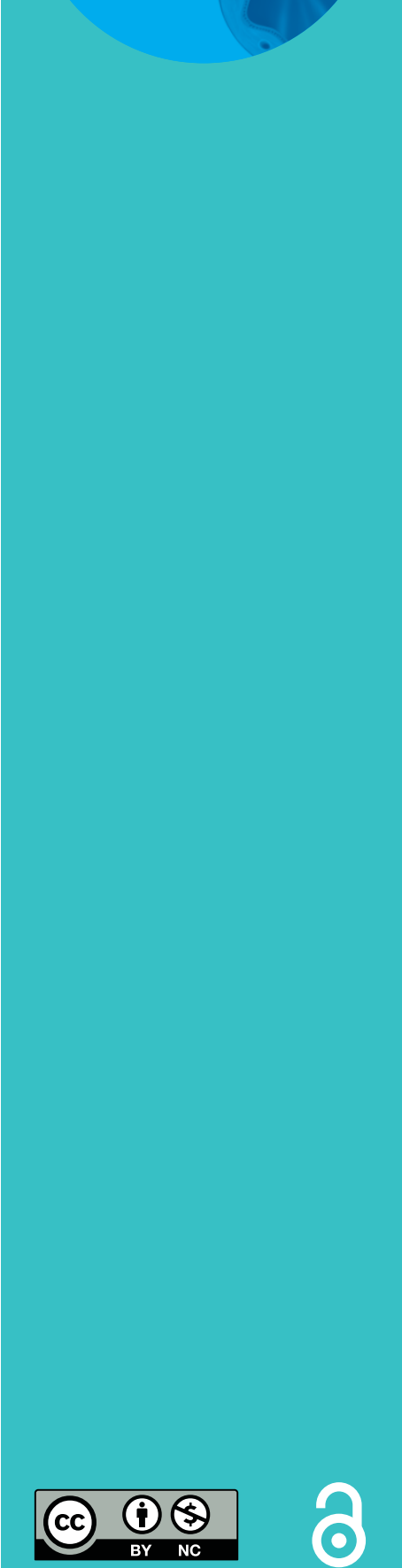

\section{Highlights from the ERS International Congress 2018: Assembly 13 - Pulmonary Vascular Diseases}

\author{
Sheila Ramjug ${ }^{1}$, Rui Adão ${ }^{2}$, Robert Lewis ${ }^{3}$, Florence Coste $\mathbb{C}^{4}$, Frances de Man ${ }^{5}$, \\ David Jimenez $\mathbb{1}^{6}$, Olivier Sitbon $\mathbb{1}^{7}$, Marion Delcroix $\mathbb{( 1}^{8}$ and \\ Anton Vonk-Noordegraaf ${ }^{5}$
}

\begin{abstract}
Affiliations: 'Dept of Respiratory Medicine, Manchester University NHS Foundation Trust, Wythenshawe, UK. ${ }^{2}$ Dept of Surgery and Physiology, Cardiovascular Research and Development Center - UnIC, Faculty of Medicine of the University of Porto, Porto, Portugal. ${ }^{3}$ PVDU, Royal Hallamshire Hospital, Sheffield, UK. ${ }^{4}$ University Bordeaux, Centre de Recherche Cardio-thoracique de Bordeaux U1045, Bordeaux, France. ${ }^{5} \mathrm{VU}$ University Medical Center, Dept of Pulmonary Medicine, Amsterdam, The Netherlands. ${ }^{6}$ Respiratory Dept, Ramon y Cajal Hospital, IRYCIS, Alcaia Henares University, Madrid, Spain. ${ }^{7}$ Pulmonary Division, Hospital Bicetre, Paris, France. ${ }^{8}$ Pneumology Dept, Universitarie Ziekenhuizen, Leuven, Belgium.
\end{abstract}

Correspondence: Sheila Ramjug, Manchester University NHS Foundation Trust, Southmoor Road, Wythenshawe. M23 9LT. E-mail: sheilaramjugahotmail.com

ABSTRACT The 2018 European Respiratory Society (ERS) International Congress in Paris, France, highlighted the subject of pulmonary vascular disease (PVD). 2018 was an exciting year for the PVD community as it was the first ERS International Congress since the formation of Assembly 13, which is dedicated to PVD, pulmonary embolism and the right ventricle.

This article aims to summarise the high-quality studies presented at the 2018 Congress into four subject areas: the use of risk stratification in pulmonary arterial hypertension, the molecular mechanisms and treatment of pulmonary hypertension $(\mathrm{PH})$, understanding and improving the right ventricle in $\mathrm{PH}$, and finally, advances in the field of acute pulmonary embolus.

@ERSpublications

This article aims to summarise the research presented at \#ERSCongress 2018: risk stratification in PAH, the molecular mechanisms and treatment of $\mathrm{PH}$, understanding and improving the RV in $\mathrm{PH}$, and advances in the field of acute pulmonary embolus http://ow.ly/WUcR30nFEcM

Cite this article as: Ramjug S, Adão R, Lewis R, et al. Highlights from the ERS International Congress 2018: Assembly 13 - Pulmonary Vascular Diseases. ERJ Open Res 2019; 5: 00202-2018 [https://doi.org/10.1183/23120541.00202-2018].

Received: Oct 272018 | Accepted after revision: Jan 252019

Copyright $\odot$ ERS 2019. This article is open access and distributed under the terms of the Creative Commons Attribution Non-Commercial Licence 4.0. 


\section{Risk stratification in pulmonary arterial hypertension}

Risk stratification in pulmonary arterial hypertension (PAH) was a topic of significant interest at the 2018 European Respiratory Society (ERS) International Congress, which included a dedicated oral presentation session on this subject.

The first paper to conceptualise risk in PAH was published by the US National Institutes of Health (NIH) 1991 [1]. The registry reported on a relatively small number of patients (194 recruited from multiple centres) at a time before pulmonary hypertension $(\mathrm{PH})$ was defined beyond primary and secondary. This study identified factors conferring higher risk and derived a risk equation consisting of three variables: mean pulmonary artery pressure (mPAP), mean right atrial pressure (mRAP) and cardiac index. The NIH registry was a landmark paper at the time that recognised the importance of right ventricular (RV) dysfunction and has been utilised by a large number of clinical trials since in determining whether treatments improve survival. In 2010, application of the equation to a cohort of 576 patients found that it underestimated life expectancy, possibly due in part to the availability of PAH-specific treatments [2].

When the 2015 European Society of Cardiology (ESC)/ERS guidelines were published, a new risk model was proposed. Thresholds for low ( $<5 \%$ 1-year mortality; "green zone"), intermediate (5-10\%; "yellow zone") and high risk (>10\%; "red zone") were created for a range of variables; the thresholds were largely based on expert opinion [3]. Three separate registries have subsequently sought to validate the "traffic light" criteria.

KYlHammar et al. [4] attempted to validate the ESC/ERS profile prognostication by a retrospective analysis of the Swedish PAH Registry, consisting of 530 patients with group $1 \mathrm{PH}$, enrolled between 2008 and 2016. Subgroups of idiopathic PAH versus associated subgroups of PAH were analysed. Variables (up to eight) were assigned a score of 1-3 based on low, intermediate or high risk. The total scores for available criteria were averaged and rounded to the nearest whole integer. There were several individually missing criteria but nonetheless, the study demonstrates that the proposed profiling strategy accurately predicts risk at both baseline and follow-up in the same group of patients. This appeared to be true of both idiopathic $\mathrm{PAH}$ and PAH related to connective tissue disease. Numbers for other subgroups of PAH were small and data are not individually presented. Of particular importance, they successfully demonstrated that achieving a low risk profile at follow-up produced the best outcomes, which suggests aggressive early treatment may be beneficial.

The COMPERA (Comparative, Prospective Registry of Newly Initiated Therapies for Pulmonary Hypertension) group took a similar methodological approach to averaging scores [5]. They focussed on six variables from the ESC/ERS guidelines: World Health Organization (WHO) functional class (FC), 6-min walk distance (6MWD), either N-terminal pro-brain natriuretic peptide (NT-proBNP) or brain natriuretic peptide (BNP), mRAP, cardiac index and mixed venous oxygen saturation.

In a large registry, 1588 patients had at least two criteria available at follow-up and $82.6 \%$ had five of the six variables recorded at baseline. Like the Swedish registry, they confirmed that the proposed thresholds appeared accurate, and that improvement between baseline and follow-up appeared critical in terms of survival.

Taking a different approach, the French registry focussed on survival benefits conferred by achieving a low risk profile. By analysing four criteria (WHO FC, 6MWD, mRAP and cardiac index), they identified that survival of patients with idiopathic, heritable or drug-induced PAH was strongly associated with the number of low-risk criteria achieved at the first follow-up visit. Patients who had a low risk profile (defined as consisting of three or four low-risk criteria) had better outcomes. Prognosis was not affected by which of the three low-risk criteria were present, suggesting that they may be similarly weighted. Achieving or maintaining a low risk profile at follow-up conferred a 1-year mortality risk of 0-1\% [6]. In addition, when BNP/NT-proBNP low-risk criteria were added in the multivariable model, only noninvasive criteria (New York Heart Association (NYHA)/WHO FC, 6MWD and BNP/NT-proBNP) were independently associated with survival, patients achieving these three criteria having an excellent long-term survival of $97 \%$ at 5 years [6]. It is worth noting that haemodynamic assessment via right heart catheterisation is necessary to confirm diagnosis and to revaluate patients who are deteriorating. This study, however, suggests that with a continued low-risk profile, noninvasive measures (NYHA/WHO FC, 6MWD and BNP/NT-proBNP) may suffice.

These validation studies were widely quoted at the 2018 ERS International Congress. Interestingly, presentations on risk from the USA also used the ESC/ERS risk stratification rather than the alternative REVEAL (Registry to Evaluate Early and Long-Term PAH Disease Management) risk calculator, which has also been retrospectively validated [7]. Both approaches currently lack prospective validation.

Several studies were presented regarding outcomes in patients with PAH due to systemic sclerosis (SSc). Mercurio et al. [8] identified that an abbreviated score (consisting of WHO FC, 6MWD, mRAP, cardiac 
index and NT-proBNP) accurately prognosticated in PAH-SSc and confirmed that patients achieving a low risk profile by first follow-up had a better prognosis.

Meanwhile, the Heidelberg group demonstrated that in patients with SSc who had borderline elevation of the pulmonary artery pressures (mPAP $20-25 \mathrm{mmHg}$ ), treatment with ambrisentan significantly improved cardiac output and pulmonary vascular resistance (PVR) but did not improve 6MWD. However, it remains unclear as to whether treatment of these patients confers any survival benefit [9].

The Ambition study, published in 2015, demonstrated that patients treated with initial combination oral therapy consisting of ambrisentan and tadalafil had better outcomes than those treated with monotherapy; this led to further development in PAH treatment $[10,11]$.

At the 2018 Congress, Boucly et al. [12] presented data from the French registry showing that patients with idiopathic PAH who were commenced on triple therapy (including either intravenous or subcutaneous prostanoid) at diagnosis had more severe haemodynamics abnormality but had better survival when compared to patients treated with mono- or dual therapy. This may suggest that patients should be treated more aggressively from the point of diagnosis, although as the majority of patients on dual therapy were receiving combination oral treatment, it is possible that the survival benefit was due to parenteral prostanoid treatment.

Selexipag, an oral prostacyclin receptor agonist, was approved for use in the USA in 2015. The Prostacyclin Receptor Agonist in Pulmonary Arterial Hypertension (GRIPHON) study demonstrated that patients receiving active treatment had a $40 \%$ risk reduction of reaching a composite primary endpoint of morbidity/mortality [13].

In work presented at the ERS Congress, SITBON et al. [14] applied the ESC/ERS risk assessment tool to participants in the GRIPHON study in a post hoc analysis. They demonstrated that patients treated with selexipag were significantly more likely to increase their number of low-risk criteria from baseline to follow-up, suggesting that treating patients with selexipag may improve the risk profile.

Rarer subtypes of PAH are often excluded from clinical trials. Patients with portopulmonary hypertension are often treated with targeted pulmonary vasodilators but there have been no published randomised controlled trials identifying optimal treatment. Early results from PORTICO (Portopulmonary Hypertension Treatment with Macitentan) were presented at the 2018 Congress, which found that patients receiving treatment had a $35 \%$ reduction in PVR, although the trial has not yet demonstrated improvement in WHO FC or 6MWD [15].

Quality of life scores are widely used in PAH. The emPHasis-10 (E10) questionnaire has been proposed as a shorter alternative to the CAMPHOR (Cambridge Pulmonary Hypertension Outcome Review) score. WORT et al. [16] demonstrated that E10 is a predictor of outcome in PAH and chronic thromboembolic $\mathrm{PH}$. It was suggested that this could be incorporated into future risk stratification algorithms. The E10 has been translated into other languages and shows excellent correlation with other quality of life scores [17].

In summary, risk stratification in $\mathrm{PAH}$ remains an area of significant interest. Changes may be made to the guidelines following the 2018 WHO symposium in Nice, France, and further research is required to prospectively validate the risk stratification models.

\section{Molecular mechanisms and treatments in PH}

Significant therapeutic advances have been observed in the field of PAH in the last decade. Although translational and basic research have already identified some interesting potential targets, patients with $\mathrm{PAH}$ and other forms of $\mathrm{PH}$ continue to have suboptimal survival and functional limitation [3], suggesting that as a PVD community, we have commenced our ascent in the understanding of the disease process; it was therefore stimulating to have sessions dedicated to translational and basic research in the field of $\mathrm{PH}$.

In PVD, it is challenging to obtain human lung parenchymal samples due to the risk of the biopsy procedure. It is therefore of paramount importance to have precise animal models. Across respiratory diseases, it is evident that animal models present some limitations, the most salient constraint being the false-positive result of a therapy. It is therefore recommended in translational research that more than one rodent model is utilised to test potential therapies/targets [18].

Worldwide, many patients with PVD fall into the category of group 3 (lung disease-related PH). Despite this, there are no specific therapies available; accordingly, it is important to find pertinent animal models. At the ERS Congress, a potential rodent model for $\mathrm{PH}$ related to pulmonary fibrosis was discussed. The team instilled intratracheal bleomycin to induce fibrosis and subsequent PH. After 3 weeks, considerable $\mathrm{PH}$ pathology was demonstrated by evidence of arterial remodelling, raised mPAP and compromised RV function [19]. 
Previous studies have also utilised bleomycin in varying concentrations and by different routes to induce $\mathrm{PH}$ related to pulmonary fibrosis. Two rodent studies either injected or instilled intratracheal bleomycin at $2.5 \mathrm{mg} \cdot \mathrm{kg}^{-1}$ and observed the effects 2 weeks later [20,21], or intratracheal bleomycin $1.5 \mathrm{mg} \cdot \mathrm{kg}^{-1}$ [22], whilst other studies injected the mice, on average, twice weekly for 4 weeks and the effects studied at 1 week thereafter $[23,24]$.

These studies have therefore exhibited the usefulness of rodent models with bleomycin-induced $\mathrm{PH}$ secondary to pulmonary fibrosis to test potential therapeutic targets, but as a community, we must ensure we also use another rodent model to confirm our findings [18].

Endothelin receptor antagonists (ERAs) are an established treatment strategy in PAH [3]. In a comparison rodent bleomycin-induced PH study of the ERAs macitentan and bosentan, macitentan significantly prevented pulmonary vascular remodelling, RV hypertrophy and cardiomyocyte size increase. It was hypothesised that the improved molecular structure of macitentan allowed superior tissue penetration [22]. In accordance with these results, preliminary work presented at the Congress suggests that transition of macitentan from bosentan is safe and efficacious in PAH patients [25].

Pirfenidone is one of the antifibrotics utilised in idiopathic pulmonary fibrosis. This antifibrotic is able to slow down the progression of fibrosis but not halt the process of fibrosis. Pirfenidone is also known for its anti-inflammatory and antiproliferative properties. An experimental animal study employing bleomycin to induce the fibrosis and $\mathrm{PH}$ revealed that in mild to moderate fibrosis, macitentan was able to ameliorate $\mathrm{PH}$ and fibrosis whilst pirfenidone appeared to be only effective against the fibrosis [26].

PoBle et al. [27], however, presented that in the Sugen5416 hypoxia rat model, the instillation of pirfenidone in this model of severe obliterative $\mathrm{PH}$ showed reduced pulmonary vascular remodelling via regulation of a forkhead box O-1 mechanism.

Many studies have also focused on the role of growth factors in PAH. Research has shown that some growth factors are implicated in the pathogenesis of PAH. Growth factors bind to their transmembrane tyrosine kinases receptors that lead to complex signalling pathways able to mediate pathobiological features in PAH, such as proliferation, migration, inflammation and apoptosis [28].

On the quest to find the prime therapeutic target, many factors have been studied. Platelet-derived growth factor is known to induce hyperproliferation of pulmonary artery smooth muscle cells [29-31], and fibroblast growth factor-2 and its production are increased in idiopathic PAH [32]. In addition, transforming growth factor (TGF)- $\beta$ is implicated in PAH $[26,33]$, as well as epidermal growth factor, which unfortunately, was found by DAHAL et al. [34] not to be a likely therapeutic target. Vascular endothelial growth factor is expressed in the vascular lesions of $\mathrm{PAH}$ [35] and finally, nerve growth factor (NGF). NGF is an important factor for the development of neurons and is produced by pulmonary cells, and has been found to have increased expression and activation in PH models [36]. Death receptors have also been implicated in the mechanism of PAH [36, 37]. This amended list demonstrates that the pathobiology of $\mathrm{PAH}$ is multicellular and complex.

DOUARD et al. [38] presented their study at the Congress, showing that NGF increases connexin 43 expression. Connexin 43, among other connexins, is paramount for the formation of gap junctions. BILLAUD

FIGURE 1 Translational research therapeutic targets. BMPR2: bone morphogenetic protein receptor II; TGF: transforming growth factor.

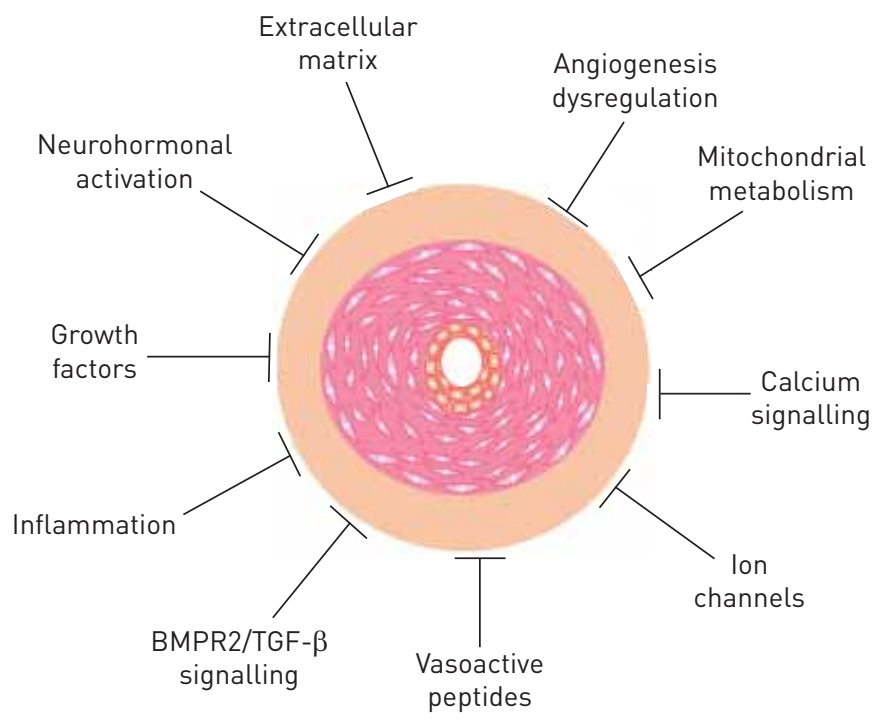


et al. [39] have already shown connexin 43 levels to be increased in $\mathrm{PH}$. The gap junctions' function is to control the communication between both smooth muscle and endothelial cells, and consequently, they have a role in the vascular response of a cell, which is important in the mechanism of PH.

Growth factors and tyrosine kinase inhibitors are promising therapeutic targets in PAH. Paradoxically, dasatinib (a tyrosine kinase inhibitor licenced for use in chronic myeloid leukaemia) is known to induce PAH [40]. Guignabert et al. [41] explained this phenomenon by conducting a comparison study between dasatinib and imatinib in rodents and humans. They discovered that dasatinib induced pulmonary endothelial cell apoptosis and pulmonary vascular damage, and it also mediated endothelial cell dysfunction via increased production of reactive oxygen species, whereas imatinib did not demonstrate these adverse effects.

A recent study by DumAs et al. [42] examined the role of $N$-methyl-D-aspartate receptors (NMDARs) in the development of PAH. NMDARs are neuronal glutamate receptors and are also expressed on vascular cells. Overexpression and overactivation of NMDARs is found in remodelled pulmonary arteries. NMDARs are activated by both endothelin-1 and by platelet-derived growth factor, which are both elevated in PAH. These conclusions propose another potential target for PAH therapy.

Translational research in $\mathrm{PH}$ and $\mathrm{PAH}$ is growing fast, allowing the discovery of many interesting targets, such as inflammation [43], growth factors [29, 30, 36], neurohormonal activation [42], the extracellular matrix [44], angiogenesis dysregulation, mitochondrial metabolism [45], bone morphogenetic protein receptor II/TGF- $\beta$ signalling [26], calcium signalling, ion channels [46, 47] ands vasoactive peptides $[48,49]$ (figure 1). Therefore, a major challenge of future translational research will be devising precise models as well as being able to prioritise drug development between these targets.

\section{Understanding and improving the RV}

The importance of the RV in PH has become more apparent with our increasing knowledge [50]. The abstracts presented at the Congress in the field of RV function provided a rich array of information pertaining to RV assessment outcomes and new clinical therapeutic interventions focused on the RV.

\section{Pulmonary hypertension}

Group I

As the pathophysiology of idiopathic PAH is an afterload mismatch, wall stress embodies the most significant feature affecting the overloaded RV. The RV mass/volume $(M / V)$ ratio has been shown to be an independent predictor of prognosis in idiopathic $\mathrm{PAH}$ [51]. RV $M / V$ ratio demonstrates the ability of the RV to adapt via concentric hypertrophy. Consequently, a higher RV $M / V$ ratio represents a more favourable adaptive process [52]; conversely, a low $M / V$ ratio can indicate either a normal or dilated RV.

In line with this, VANDERPOOL et al. [53] presented an association between $M / V$ ratio and RV function in PAH patients $(n=38)$ utilising serial measurements on PAH therapy. They found a heterogeneous association between $M / V$ ratio and afterload in this $\mathrm{PAH}$ population, suggesting that the improvement in $\mathrm{RV}$ function with combination therapy is not due to a high $M / V$ ratio of the RV. In other work, FISCHER et al. [54] demonstrated that the RV and right atrial size are independent survival predictors in PAH, representing valuable and easily accessible indicators of RV pump function during exercise, and might therefore be used for noninvasive estimation of RV contractile reserve in PAH $(n=54)$.

Sex differences in PAH and RV dysfunction severity remain a controversial topic. Women, despite being more prone to PAH development, exhibit improved survival and superior RV function than their male counterparts, suggesting oestrogens are the mediators of improved RV function [55]. As sex hormone levels change with ageing in both sexes, Groeneveld et al. [56] explored, for the first time, the effect of age on PAH disease severity and RV adaptation in both sexes $(n=298)$. RV function at baseline was not related to age in women; however, young men presented an increased afterload, and increased RV volume and mass when compared to men $>50$ years old. These noteworthy results presented at the ERS Congress suggest further research is warranted to elucidate whether this is a consequence of a distinct disease phenotype.

Tissue Doppler echocardiography (TDE) has been suggested as novel noninvasive tool to assess RV function in $\mathrm{PH}$ patients [50] and results presented at the ERS Congress support this. PFEIFFER et al. [57] presented that the overall RV TDE $(n=179)$ measurements predicted PAH and moderately correlated with prognostic parameters associated with RV function in PAH. Additionally, VRANESIC et al. [58] demonstrated that an increase in the systolic/diastolic duration ratio on echocardiography (tricuspid regurgitation Doppler) is a marker of RV dysfunction and predicts outcome in idiopathic PAH ( $\mathrm{n}=74)$.

Although the cellular mechanisms of left ventricular dysfunction have been extensively studied, the causes of RV dysfunction in $\mathrm{PAH}$ are not as well understood. Recent basic research studies, however, have 
emerged and new evidence suggests that $\mathrm{Ca}^{2+}$ mishandling contributes to RV dysfunction in PAH [59]. Recently, it was demonstrated that chronic therapy with urocortin-2 (Ucn-2), a vasoactive peptide of the corticotropin-releasing hormone family, was able to attenuate $\mathrm{PAH}$ and RV dysfunction in monocrotaline (MCT)-induced PAH animals [60, 61]. At the ERS Congress, a study by ADÃo et al. [62] extended their previous findings and tested the hypothesis that $\mathrm{Ucn}-2$ improves intracellular $\mathrm{Ca}^{2+}$ handling dysfunction in established PAH. Ucn-2 therapy failed to rescue intracellular $\mathrm{Ca}^{2+}$ handling defects but reduced the active tension development and myofilament sensitivity to $\mathrm{Ca}^{2+}$ in MCT-induced PAH rats, while acute $\mathrm{Ucn}-2$ treatment transiently and partially restored $\mathrm{Ca}^{2+}$ mishandling via stimulating protein kinase $\mathrm{A}$ and calmodulin-dependent protein kinase II.

High pulmonary pressure in uncorrected left-to-right shunt causes a progressive rise in PVR and increased perioperative mortality [63]. WALIA et al. [64] presented a retrospective analysis of children aged $\leqslant 14$ years to assess the incidence of $\mathrm{PH}$ in ventricular septal defect patients $(n=113)$ and associated post-operative outcomes of cardiac surgery. The presence of $\mathrm{PH}$ in cases of acyanotic congenital heart defects posted for surgery correlated to increased adverse perioperative outcomes and mortality. These data suggest that early surgical closure prior to development of irreversible $\mathrm{PH}$ would be desirable.

\section{Group III}

Chronic obstructive pulmonary disease (COPD) is considered a systemic disease, and the impact of the impairment of other systems and organs, such as the right heart, directly influences the prognosis of COPD patients [65]. Due the implication of oxidative stress in the development of PH and RV hypertrophy, Liu SHiu CHeOng et al. [66] tested whether allopurinol (xanthine oxidase inhibitor) reduces RV mass in PH associated with chronic lung disease $(n=71)$. Despite the lack of overall survival impact, interestingly, allopurinol reduced RV mass (allopurinol $-6.16 \mathrm{~g}$ versus placebo $0.75 \mathrm{~g}$; mean difference $6.92 \mathrm{~g}(95 \%$ CI $1.14-12.69 \mathrm{~g}), \mathrm{p}=0.02)$ in 20 COPD patients with more severe airflow limitation.

Furthermore, it is fundamental to supplement the management of COPD patients with precise methods of assessment of RV structure and function [65]. CHERnEva et al. [67] demonstrated that in patients with nonsevere COPD without PH $(\mathrm{n}=104)$, where there are limited data, right-sided cardiac structural changes are associated with lower 6MWD and stress-induced RV diastolic dysfunction. These findings may indicate that echocardiographic evidence of pulmonary vasculopathy is present in nonsevere COPD patients and contributes to decreased physical activity.

High-altitude $\mathrm{PH}$ affects individuals residing at altitudes of $\geqslant 2500 \mathrm{~m}$, and recent studies have suggested pathophysiological interactions between pulmonary haemodynamics and sleep apnoea [68]. At the ERS Congress, Muratali uUlu et al. [69] presented an interesting evaluation of RV function in highlanders with sleep-related respiratory disturbance (36 males) from Sary-Mogol (Alay region, Kyrgyzstan; 3000 m). Highlanders with apnoea-hypopnoea index $>15$ events per h had significantly increased RV Tei index and decreased $S^{\prime}$ velocity, which can point to modulation of RV failure and latent RV systolic dysfunction in these patients.

Due to the central role that RV function plays in $\mathrm{PH}$ and the fact that RV failure remains the most common cause of death in $\mathrm{PH}$ patients [70], great consideration was given at the ERS Congress to the development of means for assessing the presence/extent of RV failure, both subclinical and overt failure, as well as strategies to support the RV in $\mathrm{PH}$ and new molecular insights.

\section{Pulmonary embolism and what we need to know}

Pulmonary embolism was a hot topic at the 2018 Congress. It is well established that there is great heterogeneity in the presentation of a patient with suspected acute pulmonary embolism (PE). Over the last decade, we have seen an improvement in the outcomes of patients presenting with acute PE, hypothesised to be partly due to the advent of $\mathrm{PE}$ response teams and the appropriate use of risk stratification.

\section{Risk stratification scores}

The Pulmonary Embolism Severity Index (PESI) was initially developed and validated in 2005 [71]. In a study cohort of 15752 patients with a confirmed PE, the aim was to assess the risk of mortality and adverse events from a PE over 30 days. The PESI accurately categorised patients into classes of increasing mortality and complicated clinical course after an acute PE.

JIMÉNEZ et al. [72] calculated a Simplified PESI (sPESI) of only six parameters compared to 11 variables in the original PESI. This sPESI demonstrated a similar prognostic accuracy when compared to the PESI.

A prospective multicentre study in the Netherlands provided us with the Hestia criteria. This study's main aim was to assess the efficacy and safety of outpatient management of patients with low-risk PE by 
consolidated outcomes from smaller cohort studies assessing the efficacy and safety of outpatient management of patients with low-risk PE [73].

Patients were risk stratified within $24 \mathrm{~h}$ by the simple use of 11 questions and if any of the measures were found to be positive, this excluded the patient from outpatient management. Of the 297 patients included in this study and followed up over 3 months, there were three fatalities, none of which were due to PEs.

\section{Low-risk PEs}

In 2018, the British Thoracic Society (BTS) published guidelines concerning the initial outpatient management of PE [74]. It has been established that $37-44 \%$ of PEs could be managed in the outpatient setting but there appeared to be significant variability among centres [75].

In a Hot Topic session on Monday, September 17, Dr Howard informed the Congress that the BTS guideline recommends the use of the clinically validated scores (the Hestia criteria (table 1) [73], PESI [71] and sPESI [72]) to stratify risk in patients in the initial phase.

Individuals with low risk, i.e. PESI I/II, sPESI 0 and meeting the Hestia criteria, should be considered for outpatient management, with the provison that if sPESI or PESI is used to stratify the patient, then a set of exclusion criteria should also be applied.

\section{Intermediate-risk PEs}

In another Hot Topic session, Prof. Sanchez described that with further clinical experience and use of risk stratification tools, it has become apparent that a subset of normotensive patients in the intermediate-risk group may require escalation of therapy; hence, in 2014, the Bova score (table 2) was developed to identify those patients in the intermediate group who required more intensive monitoring and potential escalation in therapy [77].

This score was derived from six studies involving 2874 normotensive patients with PE. Isolated markers of RV dysfunction and myocardial injury, such as troponin, BNP and RV size on computed tomography, have proven to provide poor prognostic information alone; therefore, the Bova score offered us a multivariable prognostic model. The variables included systolic blood pressure $90-100 \mathrm{mmHg}$, tachycardia $\geqslant 110$ beats per min, raised troponin and evidence of RV dysfunction. The model identified three groups (I-III) with 30-day PE-related complication rates of 4.2\%, $10.8 \%$ and $29.2 \%$ [77].

This score was recently validated prospectively and had been extensively validated retrospectively [78]. Bova and colleagues have commented, however, that this score requires further refinement to not exclude the adverse events that occur in group II of the Bova score and intermediate risk in the ESC risk stratification tool [79].

Prof. Sanchez therefore introduced the concept of copeptin, lactate and hyponatraemia as additive prognostic markers, which could further improve risk stratification in the intermediate risk group.

\section{TABLE 1 Hestia criteria}

Haemodynamically unstable? ${ }^{\#}$

Thrombolysis or embolectomy necessary?

Active bleeding or high risk of bleeding?

Oxygen supply to maintain oxygen saturation $>90 \%$ for $>24 \mathrm{~h}$ ?

Pulmonary embolism diagnosed during anticoagulant treatment?

Intravenous pain medication $>24 \mathrm{~h}$ ?

Medical or social reason for treatment in the hospital $>24 \mathrm{~h}$ ?

Creatinine clearance of $<30 \mathrm{~mL} \cdot \mathrm{min}^{-1}$ ? $^{+}$

Severe liver impairment? $\$$

Pregnant?

Documented history of heparin-induced thrombocytopenia?

If one of the questions is answered as "yes", the patient cannot be treated at home. ": including the following criteria but left to the discretion of the investigator: systolic blood pressure $<100 \mathrm{mmHg}$ with heart rate $>100$ beats per min, condition requiring admission to an intensive care unit. ": gastrointestinal bleeding in the preceding 14 days, recent stroke $(<4$ weeks ago), recent surgery $(<2$ weeks ago), bleeding disorder or thrombocytopenia (platelet count $<75 \times 10^{9}$ per L), or uncontrolled hypertension (systolic blood pressure $>180 \mathrm{mmHg}$ or diastolic blood pressure $>110 \mathrm{mmHg}$. ${ }^{+}$: calculated creatinine clearance according to the Cockcroft-Gault formula. ${ }^{\S}$ : left to the discretion of the physician. Reproduced and modified from [76] with permission from the publisher. 


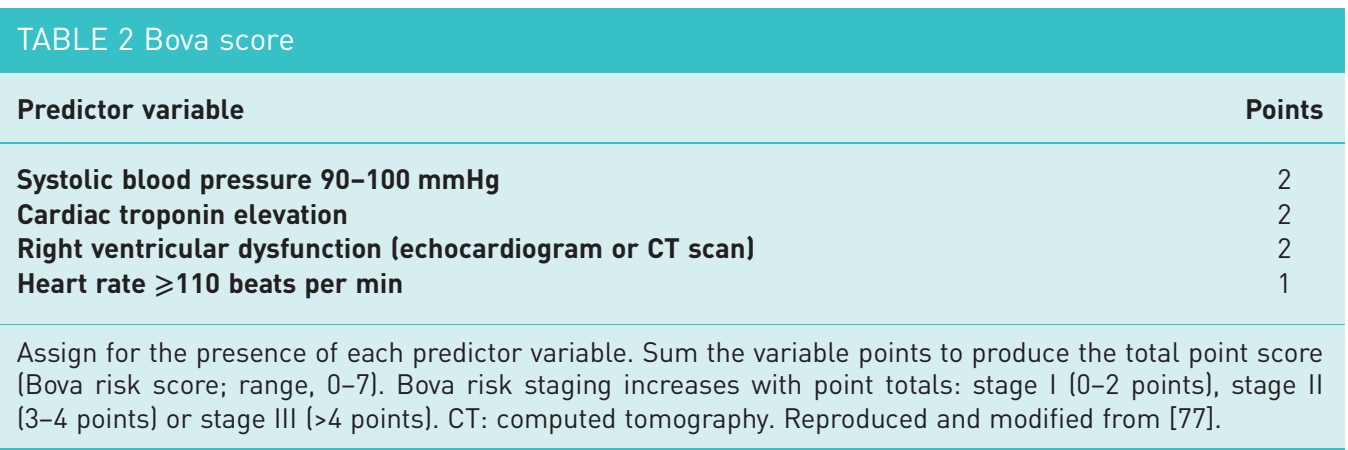

Copeptin is the C-terminal fragment of provassopressin and is found to be a useful prognostic indicator in heart failure and acute coronary syndromes. WYZGAE et al. [80] conducted a case-control study of 107 consecutive patients who presented with PE and 64 controls. PE severity was classified according to ESC guidance. Venous blood samples were drawn at admission from the participants. The study found three $(2.8 \%)$ patients with high-risk PE, intermediate risk in 69 (64.5\%) and low risk in 35 (32.7\%) patients. Copeptin levels reflected the severity of thromboembolic load and were higher in those with PE-related complications.

Hyponatraemia is a marker of neurohormonal activation, and associated with adverse outcomes in heart failure and pneumonia. Hyponatraemia was first demonstrated to be potential poor prognostic marker in acute pulmonary embolism back in 2010 [81]. A more recent, large meta-analysis using mostly retrospective cohorts has again highlighted the prognostic importance of this this inexpensive test [82]. At the Congress, this finding was also echoed by a poster by FIROOzEHI et al. [83] of a prospective study of 121 patients who found that those with hyponatraemia on admission had a significantly higher PESI score and 30-day mortality.

Other promising serum predictive markers included lactate [84] and heart-type fatty acid binding protein (H-FABP). H-FABP is a marker of early myocardial injury and in a post hoc analysis of the PROTECT study (Prognostic Value of CT Scan in Haemodynamically Stable Patients), H-FABP was found to offer improved confidence of a low-risk course after acute PE if the H-FABP was normal and in those participants classed as intermediate-high risk, an elevated H-FABP was associated with at a 12-fold increased risk of a complicated clinical course compared to those in the low-risk category [85].

\section{Advanced therapies for intermediate-high-risk PEs}

In the same Hot Topic session, Prof. Lankeit reinforced that haemodynamically unstable patients who present with a high-risk acute PE die from RV failure [86]. Systemic thrombolysis is presently the gold standard in such high-risk groups. Conversely, the use of systemic thrombolysis in the intermediate-high-risk group is limited due to the potentially catastrophic bleeding risks associated with it. Accordingly, alternative means of thrombolysis have been investigated.

The use of ultrasound catheter-directed thrombolysis (CDT) has proven popular [87]. The incidence of intracranial bleeds with CDT is lower; however, there is still a significant risk of major bleeds predominantly at the access site. These adverse events appear to correlate with the use of inferior vena cava filters as well as the burden of PE [88].

Given the still significant major bleeding risk, the recently published OPTALYSE PE randomised controlled trial demonstrated that intermediate-risk group patients treated with CDT using a shorter delivery duration and lower dose of thrombolysis still had improved RV function and clot burden when compared to the standard delivery regime and two other reduced-dose groups [89].

The PEITHO trial originally involved 76 centres and compared the use of tenecteplase with placebo for the thrombolysis of patients in the intermediate-risk group. This trial did demonstrate that those patients who received thrombolysis had a reduced incidence of death and haemodynamic collapse in the first 7 days but this initial reduction does not convey any survival benefit between the two groups at 30 days. In addition, the thrombolysis group was at increased risk of intracerebral haemorrhage (2\%) and major bleeding (6.3\%) [90].

The long-term follow-up of participants in the PEITHO trial revealed that thrombolytic therapy in the intermediate group appears to have little effect on symptoms of dyspnoea and RV function. This was a thought-provoking finding by the extension study of the PEITHO trial [91]. 
Direct oral anticoagulant agents

Also in the Hot Topic session, Prof. Becattini informed the Congress on the use of direct oral anticoagulants (DOACs). DOACs may offer the clinician a noninferior alternative to vitamin $\mathrm{K}$ antagonists with less bleeding risk [92].

Some DOACs also offer a quicker time to therapeutic anticoagulation and options such as rivaroxaban [93] or apixaban [94] require no low molecular weight heparin (LMWH) bridging, making it an attractive single-drug regime for patients.

The optimal treatment of cancer-related PEs awaits the results of three large randomised controlled trials: the Canvas trial, the Caravaggio study and the Adam VTE trial. Nevertheless, two large studies have demonstrated the noninferiority of DOACs, namely edoxaban [95] and rivaroxaban [96], when compared to LMWH. It was noted, however, that there was a higher risk of bleeding with DOACs in comparison to LMWH.

When edoxaban was compared to LMWH, the rates of major bleeding (category 2: clinically relevant nonmajor bleeding) was significantly higher $(6.9 \%$ versus $4.0 \%, \mathrm{p}=0.04)$. This was predominantly due to upper gastrointestinal (GI) bleeding with edoxaban in participants with GI malignancy. Importantly, when comparing the risk of severe major bleeding (category 3-4) between edoxaban and LMWH, there were no significant differences.

These findings were also echoed by a recent meta-analysis that ascertained that DOACs were related to higher rates of nonmajor bleed, possibly due to the type of malignancy and the potential interaction of other drugs affecting its bioavailability [97].

Subsequently, bleeding risk scores for anticoagulants have been developed [98]. The most promising is the venous thromboembolism bleed score but this requires prospective validation [99].

\section{Follow-up of patients after an acute PE}

Follow-up recommendations vary amongst the studies performed. Currently, the BTS suggests that in the first 7 days, there should be a formal review [74]. The Hestia group arranged face-to-face consultation at 1 and 12 weeks with a telephone consultation at 6 weeks [73]. An American study using a DOAC in the outpatient setting arranged a telephone consultation in the first $24-48 \mathrm{~h}$ followed by an appointment at 6 weeks and then between 12-24 weeks [100].

There is, however, a paucity of data regarding the optimum duration of follow-up and the benefit of dedicated PE clinics, as few European centres offer this service in the long-term. The PETHIO post hoc analysis implies that a third of patients appear to have continued post-PE impairment at 2 years and this can be determined by the absence of echocardiographic recovery at 6 months post-event. This conclusion will perhaps help us to shape improved long-term follow-up of our acute PE patients.

\section{Concluding remarks}

The 2018 ERS Congress has highlighted the advances in the field of PVD and provides us with a strong foundation to build upon in the coming years. At this year's Congress, we look forward to showcasing further innovative research in the field.

Conflict of interest: S. Ramjug has nothing to disclose. R. Adão has nothing to disclose. R. Lewis has nothing to disclose. F. Coste has nothing to disclose. F. de Man reports grants from the Netherlands CardioVascular Research Initiative and VENI from the Netherlands Organization for Scientific Research, outside the submitted work. D. Jimenez has nothing to disclose. O. Sitbon reports grants, personal fees and non-financial support from Actelion, Bayer, GSK and Merek, and personal fees from Acceleron and Arena, outside the submitted work. M. Delcroix reports grants and personal fees from Actelion, and personal fees from Bayer, GSK, MSD, Reata, Bellarophon and Eli Lilly, outside the submitted work. A. Vonk-Noordegraaf reports personal fees from Actelion and Arena, and grants from the Netherlands CardioVascular Research Initiative, outside the submitted work.

\section{References}

1 D'Alonzo GE, Barst RJ, Ayres SM, et al. Survival in patients with primary pulmonary hypertension. Results from a national prospective registry. Ann Intern Med 1991; 115: 343-349.

2 Thenappan T, Shah SJ, Rich S, et al. Survival in pulmonary arterial hypertension: a reappraisal of the NIH risk stratification equation. Eur Respir J 2010; 35: 1079-1087.

3 Gailè N, Humbert M, Vachiéry J-L, et al. 2015 ESC/ERS Guidelines for the diagnosis and treatment of pulmonary hypertension. Eur Respir J 2015; 46: 903-975.

4 Kylhammar D, Kjellström B, Hjalmarsson C, et al. A comprehensive risk stratification at early follow-up determines prognosis in pulmonary arterial hypertension. Eur Heart J 2017; 40: 596.

5 Hoeper MM, Pan Z, Eichstaedt CA, et al. Mortality in pulmonary arterial hypertension: prediction by the 2015 European pulmonary hypertension guidelines risk stratification model. Eur Respir J 2017; 50: 1700740. 
Boucly A, Weatherald J, Savale L, et al. Risk assessment, prognosis and guideline implementation in pulmonary arterial hypertension. Eur Respir J 2017; 50: 1700889.

7 Benza RL, Gomberg-Maitland M, Miller DP, et al. The REVEAL Registry risk score calculator in patients newly diagnosed with pulmonary arterial hypertension. Chest 2012; 141: 354-362.

8 Mercurio V, Diab N, Peloquin G, et al. Risk assessment in scleroderma patients with newly diagnosed pulmonary arterial hypertension: application of the ESC/ERS risk prediction model. Eur Respir J 2018; 52: 1800497.

9 Pan Z, Eichstaedt CA, Benjamin N, et al. Early Treatment of Borderline Pulmonary Arterial Hypertension Associated With Systemic Sclerosis (SScAPAH) (EDITA). Eur Respir J 2018; 52: Suppl. 62, OA272.

10 Galiè N, Barberà JA, Frost AE, et al. Initial use of ambrisentan plus tadalafil in pulmonary arterial hypertension. N Engl J Med 2015; 373: 834-844.

11 Rivera-Lebron BN, Risbano MG. Ambrisentan: a review of its use in pulmonary arterial hypertension. Ther Adv Respir Dis 2017; 11: 233-244.

12 Boucly A, Savale L, Weatherald J, et al. Impact of initial treatment strategy on long-term survival in pulmonary arterial hypertension (PAH). Eur Respir J 2018; 52: Suppl. 62, OA271.

13 Sitbon O, Channick R, Chin KM, et al. Selexipag for the treatment of pulmonary arterial hypertension. $N$ Engl $J$ Med 2015; 373: 2522-2533.

14 Sitbon O, Chin K, Channick RN, et al. Risk assessment in pulmonary arterial hypertension (PAH): insights from the GRIPHON study. Eur Respir J 2018; 52: Suppl. 62, OA268.

15 Sitbon O, Bosch J, Cottreel E, et al. Efficacy and safety of macitentan in portopulmonary hypertension: the PORTICO trial. Eur Respir J 2018; 52: Suppl. 62, OA267.

16 Wort S, Favoccia C, Kempny A, et al. emPHasis-10 score predicts mortality in patients with pulmonary hypertension. Eur Respir J 2018; 52: Suppl. 62, OA273.

17 Odevoglu P, Demir R, Okumuş G, et al. Evaluation of the validity and reliability of the Turkish translation of the Emphasis-10 questionnaire in patients with pulmonary hypertension. Eur Respir J 2018; 52: Suppl. 62, PA3113.

18 Bonnet S, Provencher S, Guignabert C, et al. Translating research into improved patient care in pulmonary arterial hypertension. Am J Respir Crit Care Med 2017; 195: 583-595.

19 Riberio S, Lecocq M, De Beukelaer M, et al. Development of an animal model for group 3 pulmonary hypertension. Eur Respir J 2018; 52: Suppl. 62, OA3277.

20 Rathinasabapathy A, Bruce E, Espejo A, et al. Therapeutic potential of adipose stem cell-derived conditioned medium against pulmonary hypertension and lung fibrosis. Br J Pharmacol 2016; 173: 2859-2879.

21 Shenoy V, Ferreira AJ, Qi Y, et al. The angiotensin-converting enzyme 2/angiogenesis-(1-7)/Mas axis confers cardiopulmonary protection against lung fibrosis and pulmonary hypertension. Am J Respir Crit Care Med 2010 182: 1065-1072.

22 Iglarz M, Landskroner $\mathrm{K}$, Bauer $\mathrm{Y}$, et al. Comparison of macitentan and bosentan on right ventricular remodeling in a rat model of non-vasoreactive pulmonary hypertension. J Cardiovasc Pharmacol 2015; 66: 457-467.

23 Rathinasabapathy A, Bryant AJ, Suzuki T, et al. rhACE2 therapy modifies bleomycin-induced pulmonary hypertension via rescue of vascular remodeling. Front Physiol 2018; 9: 2661.

24 Bryant AJ, Carrick RP, McConaha ME, et al. Endothelial HIF signaling regulates pulmonary fibrosis-associated pulmonary hypertension. AJP Lung Cell Mol Physiol 2016; 310: L249-L262.

25 Saleemi SA, Aldalaan AM, Weheba IM. Clinical efficacy and safety of macitentan transitioned from bosentan in PAH (an observational pilot study). Eur Respir J 2018; 52: Suppl. 62, OA3279.

26 Bellaye P-S, Yanagihara T, Granton E, et al. Macitentan reduces progression of TGF- $\beta 1$-induced pulmonary fibrosis and pulmonary hypertension. Eur Respir J 2018; 52: 1701857.

27 Poble PB, Phan C, Quatremare T, et al. Pirfenidone protects against pulmonary hypertension in the Sugen5416/ hypoxia rat model. Eur Respir J 2018; 52: Suppl. 62, OA274.

28 Peplow PV. Influence of growth factors and cytokines on angiogenic function of endothelial progenitor cells: a review of in vitro human studies. Growth Factors 2014; 32: 83-116.

29 Qian Z, Li Y, Yang H, et al. PDGFBB promotes proliferation and migration via regulating miR-1181/STAT3 axis in human pulmonary arterial smooth muscle cells. AJP Lung Cell Mol Physiol 2018; 315: L965-L976.

30 Perros F, Montani D, Dorfmüller P, et al. Platelet-derived growth factor expression and function in idiopathic pulmonary arterial hypertension. Am J Respir Crit Care Med 2008; 178: 81-88.

31 Zaiman AL, Podowski M, Medicherla S, et al. Role of the TGF- $\beta /$ Alk5 signaling pathway in monocrotalineinduced pulmonary hypertension. J Clin Invest 2008; 115: 2811-2821.

32 Tu L, Dewachter L, Gore B, et al. Autocrine fibroblast growth factor-2 signaling contributes to altered endothelia phenotype in pulmonary hypertension. Am J Respir Cell Mol Biol 2011; 45: 311-322.

33 Zaiman AL, Podowski M, Medicherla S, et al. Role of the TGF- $\beta /$ Alk5 signaling pathway in monocrotalineinduced pulmonary hypertension. Am J Respir Crit Care Med 2008; 177: 896-905.

34 Dahal BK, Cornitescu T, Tretyn A, et al. Role of epidermal growth factor inhibition in experimental pulmonary hypertension. Am J Respir Crit Care Med 2010; 181: 158-167.

35 Al-Husseini A, Kraskauskas D, Mezzaroma E, et al. Vascular endothelial growth factor receptor 3 signaling contributes to angioobliterative pulmonary hypertension. Pulm Circ 2015; 5: 101-116.

36 Freund-Michel V, Cardoso Dos Santos M, Guignabert C, et al. Role of nerve growth factor in development and persistence of experimental pulmonary hypertension. Am J Respir Crit Care Med 2015; 192: 342-355.

37 Hameed AG, Arnold ND, Chamberlain J, et al. Inhibition of tumor necrosis factor-related apoptosis-inducing ligand (TRAIL) reverses experimental pulmonary hypertension. J Exp Med 2012; 209: 1919-1935.

38 Douard M, Robillard P, Deweirdt J, et al. NGF induces pulmonary arterial hyperreactivity through increased connexin-43 expression: potential role in pulmonary hypertension. Eur Respir J 2018; 52: Suppl. 62, OA3274.

39 Billaud M, Dahan D, Marthan R, et al. Role of the gap junctions in the contractile response to agonists in pulmonary artery from two rat models of pulmonary hypertension. Respir Res 2011; 12: 30 .

40 Weatherald J, Chaumais M-C, Savale L, et al. Long-term outcomes of dasatinib-induced pulmonary arterial hypertension: a population-based study. Eur Respir J 2017; 50: 1700217.

41 Guignabert C, Phan C, Seferian A, et al. Dasatinib induces lung vascular toxicity and predisposes to pulmonary hypertension. J Clin Invest 2016; 126: 3207-3218. 
Dumas SJ, Bru-Mercier G, Courboulin A, et al. NMDA-type glutamate receptor activation promotes vascular remodeling and pulmonary arterial hypertension. Circulation 2018; 137: 2371-2389.

43 Dumas S, Nakhleh M, Montani D, et al. Immune repertoire-based signatures in pre-capillary pulmonary hypertension. Eur Respir J 2018; 52: Suppl. 62, PA3932.

44 Collum S, Weng T, Hernandez A, et al. Alternative polyadenylation (APA) modulates extracellular matrix deposition in pulmonary hypertension. Eur Respir J 2018; 52: Suppl. 62, LSC-1044.

Boucherat O, Peterlini T, Bourgeois A, et al. Mitochondrial HSP90 accumulation promotes vascular remodeling in pulmonary arterial hypertension. Am J Respir Crit Care Med 2018; 198: 90-103. development of pulmonary arterial hypertension. Circulation 2016; 133: 1371-1385.

47 Lambert M, Boet A, Rücker-Martin C, et al. Loss of KCNK3 is a hallmark of RV hypertrophy/dysfunction associated with pulmonary hypertension. Cardiovasc Res 2018; 114: 880-893.

48 Huertas A, Tu L, Guignabert C. New targets for pulmonary arterial hypertension: going beyond the currently targeted three pathways. Curr Opin Pulm Med 2017; 23: 377-385.

49 Bourgeois A, Omura J, Habbout K, et al. Pulmonary arterial hypertension: New pathophysiological insights and emerging therapeutic targets. Int J Biochem Cell Biol 2018; 104: 9-13.

50 Peacock AJ. Measuring the effects of treatment in patients with PAH: should we image the right ventricle? Eur Respir J 2017; 49: 1700805

51 Badagliacca R, Poscia R, Pezzuto B, et al. Right ventricular concentric hypertrophy and clinical worsening in idiopathic pulmonary arterial hypertension. J Heart Lung Transplant 2016; 35: 1321-1329.

52 Badagliacca R, Poscia R, Pezzuto B, et al. Right ventricular remodeling in idiopathic pulmonary arterial hypertension: adaptive versus maladaptive morphology. J Heart Lung Transplant 2015; 34: 395-403.

53 Vanderpool R, Wickstrom K, Desai A, et al. Right ventricular mass/volume in PAH: a double edged sword? Eur Respir J 2018; 52: Suppl. 62, OA1633.

54 Fischer L, Benjamin N, Egenlauf B, et al. Right ventricular size and contractile reserve significantly correlate in patients with pulmonary hypertension. Eur Respir J 2018; 52: Suppl. 62, PA3319.

55 Lahm T. Sex differences in pulmonary hypertension: are we cleaning up the mess? Eur Respir J 2016; 47: 390-393.

56 Groeneveldt J, Van Der Bruggen C, Trip P, et al. Young males with pulmonary arterial hypertension have a high afterload and low right ventricular ejection fraction at presentation. Eur Respir J 2018; 52: Suppl. 62, OA1637.

57 Pfeiffer S, Avian A, Douschan P, et al. Right ventricular tissue Doppler echocardiography in pulmonary hypertension. Eur Respir J 2018; 52: Suppl. 62, PA3111.

58 Vranesic II, Li W, Price L, et al. Increased right ventricular systolic:diastolic duration ratio predicts mortality in idiopathic pulmonary arterial hypertension. Eur Respir J 2018; 52: Suppl. 62, PA3318.

59 Prins KW, Thenappan T. World Health Organization group I pulmonary hypertension. Cardiol Clin 2016; 34: 363-374.

60 Adão R, Mendes-Ferreira P, Santos-Ribeiro D, et al. Urocortin-2 improves right ventricular function and attenuates pulmonary arterial hypertension. Cardiovasc Res 2018; 114: 1165-1177.

61 Adao R, Mendes-Ferreira P, Santos-Ribeiro D, et al. Urocortin-2 improves right ventricular function and attenuates experimental pulmonary arterial hypertension. Eur Respir J 2018; 52: Suppl. 62, PA3401.

62 Adão R, Kraler S, Holzer S, et al. Effects of urocortin-2 on cellular $\mathrm{Ca}^{2+}$ homeostasis in right heart failure induced by pulmonary artery hypertension. Eur Respir J 2018; 52: Suppl. 62, OA1634.

63 Pascall E, Tulloh RM. Pulmonary hypertension in congenital heart disease. Future Cardiol 2018; 14: 343-353.

64 Walia S, Puri G, Gandhi K, et al. Outcomes of surgical treatment of VSD with pulmonary hypertension. Eur Respir J 2018; 52: Suppl. 62, OA1638.

65 Tannus-Silva DGS, Rabahi MF. State of the art review of the right ventricle in COPD patients: it is time to look closer. Lung 2017; 195: 9-17.

66 Liu Shiu Cheong P, Lipworth B, Weir-Mccall J, et al. Allopurinol reduces RV mass in patients with severe COPD. Eur Respir J 2018; 52: Suppl. 62, OA1635.

67 Cherneva R, Cherneva Z, Valev D, et al. Right heart structural changes, exercise capacity and stress induced right ventricle dysfunction in non- severe COPD patients without pulmonary hypertension at rest. Eur Respir J 2018; 52: Suppl. 62, OA5159.

68 Latshang TD, Furian M, Aeschbacher SS, et al. Association between sleep apnoea and pulmonary hypertension in Kyrgyz highlanders. Eur Respir J 2017; 49: 1601530.

69 Muratali uulu K, Cholponbaeva M, Satybaldiev S, et al. The right ventricle function in highlanders with sleep-related respiratory disturbances. Eur Respir J 2018; 52: Suppl. 62, OA1636.

70 Amsallem M, Mercier O, Kobayashi Y, et al. Forgotten no more: a focused update on the right ventricle in cardiovascular disease. JACC Heart Fail 2018; 6: 891-903.

71 Aujesky D, Obrosky DS, Stone RA, et al. Derivation and validation of a prognostic model for pulmonary embolism. Am J Respir Crit Care Med 2005; 172: 1041-1046.

72 Jiménez D, Aujesky D, Moores L, et al. Simplification of the pulmonary embolism severity index for prognostication in patients with acute symptomatic pulmonary embolism. Arch Intern Med 2010; 170: 1383-1389.

73 Zondag W, Hiddinga BI, Crobach MJT, et al. Hestia criteria can discriminate high- from low-risk patients with pulmonary embolism. Eur Respir J 2013; 41: 588-592.

74 Howard LSGE, Barden S, Condliffe R, et al. British Thoracic Society Guideline for the initial outpatient management of pulmonary embolism (PE). Thorax 2018; 73: Suppl. 2, iil-ii29.

75 Jiménez D, Bikdeli B, Barrios D, et al. Management appropriateness and outcomes of patients with acute pulmonary embolism. Eur Respir J 2018; 51: 1800445.

76 Zondag W, Mos ICM, Creemers-Schild D, et al. Outpatient treatment in patients with acute pulmonary embolism: the Hestia Study. J Thromb Haemost 2011; 9: 1500-1507.

77 Bova C, Sanchez O, Prandoni P, et al. Identification of intermediate-risk patients with acute symptomatic pulmonary embolism. Eur Respir J 2014; 44: 694-703.

78 Bova C, Vanni S, Prandoni $\mathrm{P}$, et al. A prospective validation of the Bova score in normotensive patients with acute pulmonary embolism. Thromb Res 2018; 165: 107-111. 

Society of Cardiology risk stratification model. Eur Respir J 2016; 48: 780-786. embolism. J Thromb Thrombolysis 2015; 41: 563-568.

81 Scherz N, Labarère J, Méan M, et al. Prognostic importance of hyponatremia in patients with acute pulmonary embolism. Am J Respir Crit Care Med 2010; 182: 1178-1183.

82 Zhou X-Y, Chen H-L, Ni S-S. Hyponatremia and short-term prognosis of patients with acute pulmonary embolism: a meta-analysisInt J Cardiol 2017; 227: 251-256.

83 Firoozehi E, Faraji N, Abahti H, et al. The value of on-admission serum sodium level on 30-day outcome in pulmonary embolism. Eur Respir J 2018; 52: Suppl. 62, PA3120.

84 Vanni S, Viviani G, Baioni M, et al. Prognostic value of plasma lactate levels among patients with acute pulmonary embolism: the Thrombo-Embolism Lactate Outcome Study. YMEM 2013; 61: 330-338.

85 Dellas C, Lobo JL, Rivas A, et al. Risk stratification of acute pulmonary embolism based on clinical parameters, H-FABP and multidetector CT. Int J Cardiol 2018; 265: 223-228.

86 Harjola V-P, Mebazaa A, Čelutkienė J, et al. Contemporary management of acute right ventricular failure: a statement from the Heart Failure Association and the Working Group on Pulmonary Circulation and Right Ventricular Function of the European Society of Cardiology. Eur J Heart Fail 2016; 18: 226-241.

87 Kucher N, Boekstegers P, Müller OJ, et al. Randomized, controlled trial of ultrasound-assisted catheter-directed thrombolysis for acute intermediate-risk pulmonary embolism. Circulation 2014; 129: 479-486.

88 Sadiq I, Goldhaber SZ, Liu P-Y, et al. Risk factors for major bleeding in the SEATTLE II trial. Vasc Med 2017; 22: $44-50$.

89 Tapson VF, Sterling K, Jones N, et al. A randomized trial of the optimum duration of acoustic pulse thrombolysis procedure in acute intermediate-risk pulmonary embolism. JACC Cardiovasc Interv 2018; 11: 1401-1410.

90 Meyer G, Vicaut E, Danays T, et al. Fibrinolysis for patients with intermediate-risk pulmonary embolism. $N$ Engl J Med 2014; 370: 1402-1411.

91 Konstantinides S, Vicaut E, Danays T, et al. Impact of thrombolytic therapy on the long-term outcome of intermediate-risk pulmonary embolism. JAC 2017; 69: 1536-1544.

92 Vedovati MC, Germini F, Agnelli G, et al. Direct oral anticoagulants in patients with VTE and cancer. Chest 2015; 147: 475-483.

93 EINSTEIN-PE Investigators, Büller HR, Prins $\mathrm{MH}$, et al. Oral rivaroxaban for the treatment of symptomatic pulmonary embolism. N Engl J Med 2012; 366: 1287-1297.

94 Agnelli G, Büller HR, Cohen A, et al. Oral apixaban for the treatment of acute venous thromboembolism. $N$ Engl J Med 2013; 369: 799-808.

95 Raskob GE, van Es N, Verhamme P, et al. Edoxaban for the treatment of cancer-associated venous thromboembolism. N Engl J Med 2018; 378: 615-624.

96 Mantha S, Laube E, Miao Y, et al. Safe and effective use of rivaroxaban for the treatment of cancer associated venous thromboembolic disease. 2016; 43: 166-171.

97 Brunetti ND, Gesuete E, De Gennaro L, et al. Direct oral anti-coagulants compared with vitamin-K inhibitors and low-molecular-weight heparin for the prevention of venous thromboembolism in patients with cancer: a meta-analysis study. Int J Cardiol 2017; 230: 214-221.

98 Klok FA, Huisman MV, Konstantinides S, et al. Predicting anticoagulant-related bleeding in patients with venous thromboembolism: a clinically oriented review. Eur Respir J 2015; 45: 201-210.

99 Klok FA, Hösel V, Clemens A, et al. Prediction of bleeding events in patients with venous thromboembolism on stable anticoagulation treatment. Eur Respir J 2016; 48: 1369-1376.

100 Beam DM, Kahler ZP, Kline JA. Immediate discharge and home treatment with rivaroxaban of low-risk venous thromboembolism diagnosed in two U.S. emergency departments: a one-year preplanned analysis. Acad Emerg Med 2015; 22: 788-795. 